\title{
El clima motivacional del docente de Educación Física: ¿Puede afectar a las calificaciones del alumnado? Motivational climate of teaching physical education: Could it affect student grades? Javier Sevil Serrano, Alberto Aibar Solana, Ángel Abós Catalán, Luis García González Universidad de Zaragoza (España)
}

Resumen: El objetivo del estudio fue analizar la relación entre diversas variables motivacionales y la calificación académica en una unidad didáctica (UD) de Educación Física (EF). Participaron 224 estudiantes, de ocho grupos, con edades comprendidas entre los 12 y 14 años $(M=12.37$, SD = 0.64) durante 10 sesiones de salto con combas. Los instrumentos utilizados midieron la percepción del alumnado del clima motivacional (EPCM), las necesidades psicológicas básicas (BPNES), el tipo de motivación (SIMS-14) y las consecuencias afectivas de diversión y aburrimiento (SSI-EF) en la última sesión de la UD. La calificación en la UD de salto con combas fue valorada con una puntuación que podía oscilar entre 0 y 10 . Los resultados mostraron una relación significativa y positiva del clima motivacional tarea, las necesidades psicológicas básicas, la motivación autodeterminada y la diversión con la calificación académica, siendo el clima motivacional tarea el que mayor poder predictivo mostró sobre la calificación (35\% de la varianza explicada). En sentido contrario, el clima motivacional ego, la desmotivación y el aburrimiento se relacionaron de manera significativa y negativa con la calificación final en la UD. Se destaca la importancia que puede tener el profesorado de EF sobre la calificación académica de sus discentes cuando genera un clima motivacional tarea.

Palabras clave: comportamiento docente, motivación, necesidades psicológicas, rendimiento académico.

Abstract: The aim of the study was to analyze the relationship between motivational variables, and academic grades in a unit of physical education. Two hundred and twenty-four students, of eight classes, aged between 12 and 14 years $(M=12.37, S D=0.64)$ were involved in 10 skipping rope lessons. The instruments measured the perception of the students' motivational climate, basic psychological needs (BPNES), type of motivation (SIMS-14) and the affective consequences of enjoyment and boredom (SSI-EF) in the last session of the unit. The grade at unit was assessed with a score that could range from 0 to 10 . The results showed that task motivational climate, basic psychological needs, self-determined motivation and enjoyment were related significantly positively with the academic grade, being the task motivational climate which showed the greatest predictive effect (35\% of variance explained). Conversely, the ego motivational climate, amotivation and boredom were related significantly negative with the academic grade. This fact highlights the importance of the PE teachers on the students' academic grades when a task motivational climate task is emphasized. Key words: teacher behavior, motivation, psychological needs, academic achievement.

\section{Introducción}

En los últimos años, el rendimiento académico de los jóvenes ha despertado un creciente interés en la sociedad debido al fenómeno del fracaso escolar (Calero, Choi \& Waisgrais, 2010). Entre las numerosas definiciones de rendimiento académico algunos autores sostienen que este término hace referencia al nivel de conocimientos y habilidades que los estudiantes obtienen en el ámbito escolar. Dentro de todas las formas de evaluar el rendimiento académico, normalmente se utilizan las calificaciones obtenidas en las diferentes asignaturas, ya sea a través de una medida autorreportada de los estudiantes o a través de un informe remitido por el centro escolar (Sternberg, 2010). Algunos estudios han señalado que un bajo nivel de rendimiento académico en adolescentes pueden estar asociados a diversos trastornos psicológicos, como la ansiedad y la depresión (Huang, 2015). Sin embargo, un alto nivel de rendimiento académico pueden afectar positivamente a variables psicológicas como la autoestima, el auto-concepto, y el bienestar afectivo y psicológico, disminuyendo el abandono escolar (Fati-Ashtiani, Ejei, Khodapanahi \& Tarkhorani, 2007; García, Jimmefors, Mousavi, Adrianson, Rosenberg \& Archer, 2015). Por ello, el estudio de las variables que influyen sobre el rendimiento académico en todas las etapas educativas está recibiendo actualmente una constante atención (Martín, Martínez-Arias, Marchesi \& Pérez, 2008; Risso, Peralbo \& Barca, 2010).

Tradicionalmente, el rendimiento académico era percibido como una facultad individual que no estaba estrechamente relacionada con el estilo docente (Urdan \& Schoenfelder, 2006). Recientemente, ha aumentado el número de estudios que han mostrado una interacción positiva entre docentes-discentes y el rendimiento académico (Wang \& Holcombe, 2010). En la misma línea, otros autores han señalado que el clima emocional de la clase generado por el docente (i.e., la calidad de la interacciones sociales y emocionales en el binomio profesor-alumno) (Reyes, Brackett, Rivers White \& Salovey, 2012), así como el apoyo a las relaciones sociales (King, 2015) o el apoyo a la autonomía (Alivernini

Fecha recepción: 12-02-16. Fecha de aceptación: 21-07-16 Fecha recepción: 12 javier.sevil@gmail.com
\& Lucidi, 2011) puede influir en el compromiso y en el rendimiento académico del alumnado. Así, la teoría de las metas de logro (TML; Nicholls, 1989), postula que el clima motivacional generado por el docente (i.e., las claves de éxito o fracaso que definen una actividad) puede influir de manera positiva o negativa sobre diferentes consecuencias desencadenas en el aula. Es decir, la manera en la que el profesor estructure y desarrolle sus clases puede generar en el alumnado una serie de comportamientos más o menos adaptativos en el aula.

En el contexto de Educación Física (EF), el clima motivacional tarea (i.e., se valora el esfuerzo y el progreso personal del alumnado) se ha visto relacionado con consecuencias positivas de índole afectiva como la diversión (Jaakkola, Wang, Soini \& Liukkonen, 2015), comportamental como la intención de ser físicamente activo (Cecchini, Fernández-Río, \& Méndez-Giménez, 2014) y cognitiva como las estrategias de aprendizaje y el esfuerzo (Liukkonen, Barkoukis, Watt \& Jaakkolaa, 2010). En sentido contrario, el clima motivacional ego (i.e., se valora al alumnado con criterios de comparación social) en las clases de EF se relaciona con consecuencias afectivas negativas como el aburrimiento (FernándezRío, Méndez-Giménez \& Cecchini, 2014), comportamentales como la indisciplina (Moreno, Jiménez, Gil,Aspano \& Torrero, 2011) y cognitivas como la ansiedad (Coterón-López, Franco, Pérez-Tejero \& Sampedro, 2013).

De esta manera, el desarrollo de un clima motivacional concreto por parte del docente de EF parece ser un aspecto fundamental en el desarrollo del aprendizaje y del progreso inter-individual del alumnado. Esta participación activa del alumnado en su aprendizaje o la percepción de valoración de su progreso personal, elementos relacionados con el clima tarea, podrían explicar que el alumnado se implique y sienta que es valorado con criterios individuales, dando lugar a un mayor rendimiento académico. En contraste, si el alumnado percibe un clima motivacional ego podría sentir que su progreso depende de la habilidad o rendimiento de sus compañeros, no obteniendo un control interno y autoevaluación sobre su aprendizaje (Nicholls, 1992).

Demanera complementaria, la teoría de la autodeterminación(TAD; Deci \& Ryan, 1985) señala que la intervención docente del profesor de EF puedeejercer una gran influencia en las tres necesidades psicológicas básicas (i.e., autonomía, competencia y relaciones sociales), y consecuentemente, en los procesos motivacionales desencadenados en las 
clases de EF. De este modo, la TAD establece un continuo motivacional que abarca desde la motivación intrínseca hasta la desmotivación, existiendo diferentes regulaciones intermedias, que van de más a menos autoderteminación en función del motivo que las originan (i.e., regulación integrada, identificada, introyectada y externa). En este sentido, si el alumnado satisface su necesidad de tomar decisiones y adquirir responsabilidades en el aula, de sentirse eficaz y habilidoso en las tareas que desempeña y de relacionarse e integrase con su grupo de iguales puede obtener una conducta más autodeterminada o volitiva en su proceso de enseñanza-aprendizaje (Deci \& Ryan, 1985). De manera paralela, la TAD establece que los tipos de motivación más autodeterminada se relacionaban con consecuencias positivas como un mayor bienestar, aprendizaje y rendimiento académico (Niemiec \& Ryan, 2009), mientras que las formas de motivación menos autodeterminadas se relacionan con consecuencias negativas como el aburrimiento o unmenor rendimiento académico (Legault, Green-Demers \& Pelletier, 2006).

En definitiva, estos dos entramados teóricos señalan que tanto el clima motivacional generado por los docentes de EF, como las variables motivacionales, podrían estar vinculados al rendimiento académico obtenido por el alumnado (Nicholls, 1992). En esta línea, un trabajo realizado en la asignatura de matemáticas, señaló que la satisfacción de la autonomía, la motivación autodeteminada, y especialmente el esfuerzo del alumnado, influían en los resultados académicos obtenidos por los estudiantes (León, Núñez \& Liewc, 2015). Asimismo, otros estudios han señalado que el alumnado con un mayor rendimiento académico en asignaturas como lengua castellana, EF, y matemáticas presentaban una motivación escolar más alta (González \& Portoles, 2014). En este sentido, con objeto de optimizar los procesos de enseñanza-aprendizaje, parece oportuno seguir profundizando en el estudio de las variables que influyen sobre la calificación académica en cada asignatura en particular.

Concretamente, en el contexto de la EF, autores como Boiché, Sarrazin, Grouzet, Pelletier y Chanal (2008) señalaron que los perfiles más autodeterminados se relacionaban con una mayor calificación académica en una UD de gimnasia. En esta misma línea, Barkoukis, Taylor, Chanal y Ntoumanis (2014), en un estudio longitudinal de tres años, señalaron que los estudiantes y las clases con una motivación más autodeterminada obtenían una mayor calificación académica en EF, mientras que los estudiantes y las clases con una motivación menos autodeterminada conseguían peores resultados académicos.

En base a los trabajos previos, este estudio pretende analizar la relación entre el clima motivacional generado por el docente de EF y otras variables motivacionales, sobre la calificación académica obtenida por el alumnado en una UD de EF. Como primera hipótesis, se plantea que el clima motivacional tarea, la satisfacción de las necesidades psicológicas básicas, la motivación autodeterminada y la diversión se relacionarán significativa y positivamente con la calificación académica. La segunda hipótesis sostiene que el clima motivacional ego, la regulación externa, la desmotivación y el aburrimiento se relacionarán significativa y negativamente con la calificación académica.

\section{Método}

\section{Participantes}

De una muestra inicial de 233 estudiantes, participaron finalmente en el estudio 224 estudiantes (105 hombres y 119 mujeres), de ocho grupos, con edades comprendidas entre los 12 y 14 años $(M=12.37$, $D T=0.64)$. El alumnado pertenecía a $1^{\text {er }}$ curso de Educación Secundaria Obligatoria de un centro público de Huesca. Los criterios de inclusión para la selección del alumnado fueron la cumplimentación de todos los instrumentos relativos a las variables del estudio, así como la asistencia a ocho de las 10 sesiones de la UD de salto con combas. Nueve estudiantes fueron eliminados del estudio, cuatro de ellos no cumplimentaron los cuestionarios, dos estaban lesionados y tres no asistieron al número de clases establecido en los criterios de inclusión. El tamaño de los grupos oscilaba entre un mínimo de 25 y un máximo de 30 ( $M=28.31$, $D T=0.22$.

\section{Instrumentos}

Clima motivacional. Se utilizó la Escala de Percepción del Clima Motivacional (Biddle, Cury, Goudas, Sarrazin, Famose \& Durand, 1995), en su versión traducida al castellano y adaptada a la EF (EPCM; Gutiérrez, Ruiz \& López, 2011). La frase introductoria de la escala fue modificada al contenido de salto con combas «En las clases de salto con combas nuestro profesor/a de EF...». Esta escala consta de 19 ítems, agrupados en dos factores: 12 ítems miden el clima motivacional tarea (e.g., «El profesor se siente satisfecho cuando todos mejoran») y siete ítems el clima motivacional ego (e.g., «El profesor solo se ocupa de aquellos que hacen bien los ejercicios»).

Necesidades psicológicas básicas. Se utilizó la Escala de las Necesidades Psicológicas Básicas en el Ejercicio(Vlachopoulos \& Michailidou, 2006) en su versión traducida al castellano y adaptada a la EF (Moreno, González-Cutre, Chillón \& Parra, 2008). La frase inicial de la escala fue modificada al contenido de salto con combas «En las clases de salto con combas...». Consta de 12 ítems agrupados en tres factores (cuatro ítems por factor) que miden la autonomía (e.g., «Tengo la oportunidad de elegir cómo realizar los ejercicios»), la competencia (e.g., «Realizo los ejercicios eficazmente») y las relaciones sociales (e.g., «Me siento muy cómodo/a con los/as compañeros/as»).

Tipo de motivación. Se utilizó la Escala de Motivación Situacional (SIMS; Guay, Vallerand \& Blanchard, 2000) validada al castellano en el contexto educativo (EMSI; Martín-Albo, Núñez \& Navarro, 2009). La pregunta introductoria fue modificada al contenido de salto con combas «¿Por qué has participado este año en las clases de salto con combas?». La escala está compuesta por 14 ítems, agrupados en cuatro factores: cuatro ítems miden la motivación intrínseca (e.g., «Porque me sentía bien cuando la practicaba»), tres ítems miden la regulación identificada (e.g., «Porque creo que esta actividad era importante para mí»), tres ítems miden la regulación externa (e.g., «Lo he hecho por mi propio bien») y cuatro ítems la desmotivación (e.g., «Yo he participado pero no estoy seguro que valiese la pena»). Debido a que esta versión no ha sido validada en el contexto de la EF, se comprobaron las propiedades psicométricas del instrumento a través de un análisis factorial confirmatorio (AFC), testando el mismo modelo teórico presentado en la validación española de Martín-Albo, Nuñez y Navarro (2009) y comparándolo con los datos obtenidos en este estudio. Los resultados del AFC indicaron un ajuste limitado a los datos para una estructura de cuatro factores a través de los distintos índices de ajuste evaluados $(\chi 2=$ 186.58, $p$ <.001; $\chi 2$ /g.l. = 2.63; RMSEA = .08; CFI = .92; TLI = .90; $\mathrm{SRMR}=.07)$.

Diversión/satisfacción y aburrimiento. Se utilizó la Escala de Diversión Deportiva (SSI; Duda \& Nicholls, 1992), en su versión validada en el contexto español y adaptada a la EF (Baena-Extremera, GraneroGallegos, Bracho-Amador \& Pérez-Quero, 2012). El encabezado fue modificado al contenido de salto con combas «¿Cómo te lo has pasado en las clases de salto con combas en EF...?». La escala está compuesta por un total de ocho ítems, agrupados en dos factores: cinco ítems miden la diversión (e.g., «Me solía divertir en esta actividad») y tres ítems el aburrimiento (e.g., «Deseaba que las clases acabasen pronto»).

El formato de respuesta empleado en cada uno de los instrumentos de medida estaba indicado en una escala tipo Likert del 1 al 5, donde el 1 correspondía a totalmente en desacuerdo y el 5 a totalmente de acuerdo con la formulación de la pregunta.

Calificación de la UD. Tomando como sustento el marco legislativo que rige el sistema educativo en España, se utilizó para valorar la calificación escolar de la UD de salto con combas una escala de 0 a 10 (Ley Orgánica 2/2006, de 3 de mayo). En Educación Secundaria en España esta escala va asociada a una calificación cualitativa: sobresaliente ( 9 a 10), notable (7 a 8.99), bien (6 a 6.99), suficiente (5 a 5.99) e insuficiente (0 a 4.99).

La UD de salto con combas se valoró a través de una serie de 
pruebas ponderadas acorde a los criterios de evaluación del currículo vigente en la ComunidadAutónoma deAragón(Orden de 9 de mayo de 2007, BOA de 1 de junio de 2007). Se evaluó la progresión y la actitud del alumnado (15\%) a través de una autoevaluación al alumnado. De manera complementaria, el docente utilizó un registro anecdótico para verificar que la autoevaluación del alumnado se ajustaba a la actitud mostrada en el aula. Asimismo, se evaluó un trabajo teórico-práctico sobre el salto a la comba (25\%) y una coreografía grupal (60\%).

En la tabla 1, se pueden apreciar las partes evaluadas, y sus respectivos porcentajes, en cada uno de los instrumentos de evaluación utilizados en esta UD.

Tabla 1.

\begin{tabular}{|c|c|c|c|c|}
\hline $\begin{array}{c}\text { Criterio de } \\
\text { evaluación } \\
\text { (Orden de } 9 \text { de mayo } \\
\text { de 2007, BOA de } 1 \\
\text { de junio de 2007) }\end{array}$ & $\begin{array}{l}\text { Instrumentos de } \\
\text { Evaluación }\end{array}$ & Calificación & $\begin{array}{l}\text { Partes a } \\
\text { Evaluar }\end{array}$ & $\begin{array}{l}\text { Calificación } \\
\text { de cada parte }\end{array}$ \\
\hline 3 & $\begin{array}{c}\text { Trabajo de } \\
\text { salto con combas }\end{array}$ & $25 \%$ & $\begin{array}{l}\text { - Entrega individual } \\
\text { - Entrega grupal } \\
\text { - Exposición del trabajo }\end{array}$ & $\begin{array}{l}10 \% \\
10 \% \\
5 \%\end{array}$ \\
\hline 4 & $\begin{array}{l}\text { Coreografía grupal } \\
\text { (Escala de } \\
\text { clasificación) }\end{array}$ & $60 \%$ & $\begin{array}{l}\text { - Planilla de la coreografía } \\
\text { de salto con combas } \\
\text { - Valoración de la } \\
\text { coreografía (profesor) } \\
\text { - Coevaluación de la } \\
\text { coreografía (compañeros) } \\
\text { - Autoevaluación y } \\
\text { coevaluación de la } \\
\text { coreografía (grupos) }\end{array}$ & $\begin{array}{l}5 \% \\
35 \% \\
10 \% \\
10 \%\end{array}$ \\
\hline 8 & $\begin{array}{l}\text { Autoevaluación } \\
\text { alumnado y } \\
\text { registro anecdótico }\end{array}$ & $15 \%$ & $\begin{array}{c}\text { - Ficha autoevaluación de } \\
\text { la unidad de salto con } \\
\text { combas }\end{array}$ & $15 \%$ \\
\hline
\end{tabular}

\section{Diseño y procedimiento}

Se realizó un diseño descriptivo-correlacional de tipo transversa (Montero \& León, 2007). En primer lugar, se contactó con el equipo directivo del centro de enseñanza, y posteriormente con el docente de EF implicado en el estudio, para informarles de los objetivos de la investigación y pedirles su colaboración. El estudio tuvo lugar en una UD de salto con combas, integrada en la programación del docente de EF. Las 10 clases se realizaron bajo una metodología de enseñanza comprensiva (Stolz \& Pill, 2013), centrada en el juego y la resolución de problemas, con una frecuencia de dos sesiones semanales y una duración de 50 minutos cada una. La UD se construyó atendiendo a los objetivos, contenidos, competencias básicas y criterios de evaluación del currículo vigente en la Comunidad Autónoma de Aragón (Orden de 9 de mayo de 2007, BOA de 1 de junio de 2007).

Para valorar la coreografía grupal de salto con combas se grabaron todas las actuaciones en el aula. Posteriormente, en base a los criterios seleccionados para su evaluación (e.g., participación, sincronización, ajuste a la música, vestuario, etc.) y a la ponderación de los mismos, se evaluó a través de una planilla de observación, cada una de las coreografías. En la evaluación de dichas coreografías participaron dos docentes de EF con experiencia profesional. Para garantizar la fiabilidad y validez en las calificaciones se visionó cada coreografía en dos ocasiones.

Los cuestionarios se administraron en la última sesión de la UD de salto con combas, en presencia del investigador principal y en ausencia del docente de EF. Durante todo el proceso se insistió en el anonimato de las repuestas y en la importancia de contestar con sinceridad, explicando a los estudiantes que no estaban siendo evaluados. Para la toma de datos se destinó un tiempo aproximado de 15-20 minutos. Posteriormente, se obtuvieron las notas académicas de los diferentes grupos en la UD. El Comité de Ética de la Universidad de Zaragoza aprobó la realización del estudio, siguiendo las directrices de la Declaración de Helsinki (2008) con respecto al consentimiento, confidencialidad y anonimato de las respuestas. Debido a que los participantes eran menores de edad, los padres o tutores y los propios estudiantes autorizaron previamente su participación voluntaria en el estudio.

\section{Análisis estadístico de datos}

En primer lugar, se analizó la consistencia interna de cada factor mediante el coeficiente de alfa de Cronbach. Posteriormente, se obtuvieron los estadísticos descriptivos de todas las variables del estudio (me- dia y desviación típica) (VerTabla 1). Asimismo, se llevó a cabo unAFC para la Escala de Motivación Situacional. Para analizar la relación entre las calificaciones académicas y el resto de variables del estudio se realizó un análisis de correlaciones bivariadas a través del coeficiente de correlación de Pearson. Finalmente, se realizó un análisis de regresión por pasos sucesivos considerando como variable dependiente la calificación académica de la UD e independientes el clima motivacional, las necesidades psicológicas básicas, el tipo de motivación y las consecuencias afectivas de diversión y aburrimiento. Todos los cálculos se realizaron a través de los softwares estadísticos SPSS 21.0 y MPlus 7.11.

\section{Resultados}

Se obtuvo una calificación media en la UD de salto con combas de 7.56 (DT $=1.58$ ), en la cual 14 alumnos obtuvieron un suspenso (6.3\%), 15 un suficiente (6.7\%), 21 un bien (9.4\%), 127 un notable (56.7\%) y 47 un sobresaliente (21\%).

En el análisis de correlaciones (Tabla 2) se aprecia que el clima motivacional tarea, la autonomía, competencia y relaciones sociales, la motivación intrínseca, la regulación identificada y la diversión se relacionan significativa y positivamente con la calificación académica obtenida por el alumnado en la UD. En sentido contrario, el clima motivacional ego, la desmotivación y el aburrimiento se relacionan significativa y negativamente con la calificación académica.

En el análisis predictivo por pasos sucesivos (Tabla 3) se muestra que las calificaciones académicas son predichas positivamente de manera significativa por el clima motivacional tarea y por la satisfacción de competencia. Ambas variables explicaron un 36\% de la varianza, contribuyendo de forma más elevada el porcentaje explicado por el clima motivacional tarea (35\%).

\begin{tabular}{|c|c|c|c|c|c|}
\hline \multicolumn{6}{|c|}{$\begin{array}{c}\text { Análisis descriptivo, análisis de fiabilidad y análisis de correlaciones entre la calificación en la } \\
\text { UD y el resto de variables motivacionales del estudio }\end{array}$} \\
\hline & & $D T$ & & \\
\hline Clima tarea & & 4.49 & 0.46 & .81 & $.59 * *$ \\
\hline $\begin{array}{l}\text { Clima tarea } \\
\text { Clima ego }\end{array}$ & & 2.75 & 0.60 & .80 & $-.14 *$ \\
\hline \multicolumn{2}{|l|}{ Autonomía } & 3.75 & 0.80 & .78 & $.18^{*}$ \\
\hline \multicolumn{2}{|l|}{ Competencia } & 4.04 & 0.66 & .72 & $.32 * *$ \\
\hline \multicolumn{2}{|l|}{ Relaciones sociales } & 4.36 & 0.66 & .77 & $.21^{* *}$ \\
\hline \multicolumn{2}{|l|}{ Motivación intrínseca } & 4.08 & 0.96 & .87 & $.20^{* * *}$ \\
\hline \multicolumn{2}{|l|}{ Regulación identificada } & 3.63 & 0.96 & .69 & $.20^{* * *}$ \\
\hline \multicolumn{2}{|l|}{ Regulación externa } & 3.37 & 1.26 & .82 & -.073 \\
\hline \multicolumn{2}{|l|}{ Desmotivación } & 1.85 & 0.93 & .76 & $-.23 * *$ \\
\hline \multicolumn{2}{|l|}{ Diversión/Satisfacción } & 4.25 & 0.87 & .72 & $.21^{*}$ \\
\hline \multicolumn{2}{|l|}{ Aburrimiento } & 1.77 & 0.86 & .89 & $-.13 *$ \\
\hline \multicolumn{2}{|l|}{ Rendimiento } & 7.56 & 1.58 & & \\
\hline \multicolumn{6}{|c|}{ Nota: ${ }^{*} p<.05,{ }^{* *} p<.01 . \mathrm{r}=$ coeficiente de correlación de Pearson. } \\
\hline \multicolumn{6}{|c|}{ Tabla 3.} \\
\hline \multicolumn{6}{|c|}{$\begin{array}{l}\text { Análisis de regresión de la calificación en la UD a través del resto de variables del estudio } \\
\text { Variables }\end{array}$} \\
\hline \multicolumn{6}{|l|}{ Calificación } \\
\hline Paso 1 & & & & & $.350 * *$ \\
\hline $\begin{array}{l}\text { Clima motivacional } \\
\text { tarea }\end{array}$ & 2.020 & & .185 & .592 & \\
\hline Paso 2 & & & & & $.365 * *$ \\
\hline $\begin{array}{l}\text { Clima motivacional } \\
\text { tarea }\end{array}$ & 1.866 & & .196 & .547 & \\
\hline $\begin{array}{l}\text { Satisfacción de } \\
\text { competencia }\end{array}$ & .300 & & .128 & 128 & \\
\hline
\end{tabular}

\section{Discusión}

Tomando como referencia la TML (Nicholls, 1989) y la TAD (Deci \& Ryan, 1985), el objetivo del presente estudio fue analizar la relación entre el clima motivacional generado por el docente, así como una serie de variables motivacionales, y la calificación académica obtenida en una UD de salto con combas en EF.

La primera hipótesis planteó que el clima motivacional tarea, la satisfacción de las necesidades psicológicas básicas, la motivación autodeterminada y la diversión se relacionarían significativa y positivamente con la calificación académica obtenida en dicho contenido, confirmándose a raíz de los resultados encontrados. En esta misma línea, un estudio reciente en el contexto universitario mostró la importancia del apoyo a la autonomía generado por el docente universitario en la calificación media obtenida por el alumnado (Moreno-Murcia, Silveira \& Alias, 2015). En el presente estudio, las tres necesidades psicológicas 
básicas, especialmente la percepción de competencia del alumnado, se relacionaron de manera positiva con la calificación académica obtenida por los estudiantes en la UD de salto con combas. En sintonía con los resultados encontrados, Ulstad, Halvari, Sørebø y Deci (2016) señalaron la importancia de las necesidades psicológicas básicas en el rendimiento académico obtenido en las clases de EF. Una posible justificación a los resultados encontrados en el presente estudio puede deberse a que si el alumnado se percibe competente en EF, estará más motivado e implicado para alcanzar los diferentes objetivos propuestos (Moreno-Murcia, Cervelló, Montero, Vera \& García-Calvo, 2012). Además, los resultados confirman la importancia de las formas de motivación más autodeterminada en la calificación académica obtenida por el alumnado. Estos resultados están en consonancia con los estudios de Boiché et al. (2008) y Barkoukis et al. (2014), en el contexto de la EF, que señalaron la relación entre la formas de motivación más autodeterminadas y el rendimiento académico. Por último, la relación positiva entre la diversión y la calificación académica, está en la línea de otros estudios previos (Bobbit, 2003).

De manera complementaria, los resultados obtenidos en el análisis de regresión señalaron que el clima motivacional tarea fue la variable que obtuvo un mayor poder predictivo de la calificación académica. Sin embargo, el resto de variables motivacionales integradas dentro de la TAD, salvo la percepción de competencia, no fueron predictores sobre la calificación académica en esta UD. Estos resultados están en sintonía de los postulados de la TML (Nicholls, 1989) que señalan que la percepción del alumnado de un clima motivacional tarea está relacionado con consecuencias positivas cognitivas como el esfuerzo, la atención y las estrategias de aprendizaje. En este sentido, el clima motivacional generado por el docente podría ejercer una influencia más directa sobre la calificación académica obtenida que otras variables motivacionales. Este hecho puede deberse a que la EF escolar conlleva una calificación académica en el alumnado. Por ello, si el docente valora en la calificación de la UD el progreso personal, los discentes podrían esforzarse más para obtener una mayor calificación, independientemente de su nivel de habilidad o motivación hacia el contenido. Del mismo modo, Bobbit (2003) señaló la importancia del clima motivacional sobre el aprendizaje y el rendimiento académico obtenido por los discentes en la asignatura de ciencias. De este modo, si el docente pone un mayor énfasis en la progresión inter-individual del alumnado, premiando los éxitos conseguidos, cediendo decisiones y responsabilidad a lo largo de las diferentes sesiones, el alumnado puede percibir que su esfuerzo se está viendo recompensado. En esta misma línea, algunos autores han señalado que el esfuerzo del alumnado está relacionado con un mayor rendimiento académico (Federici \& Skaalvik, 2014). Por tanto, los resultados sugieren que el profesorado de EF puede ejercer, en el diseño y la propia intervención docente, un papel trascendental para que el alumnado se esfuerce y trate de progresar y sentirse competente en su aprendizaje, obteniendo unas mejores calificaciones académicas.

La segunda hipótesis sostuvo que el clima motivacional ego, la regulación externa, la desmotivación y el aburrimiento se relacionarín significativa y negativamente con la calificación académica. En líneas generales, los resultados encontrados apoyan la segunda hipótesis. De manera consistente con el estudio de Bobbit(2003), el clima motivacional ego generado por el docente parece ser un factor que está relacionado con un menor rendimiento académico en esta UD. Por tanto, parece que la construcción de un clima motivacional en el que el docente de EF evite la comparación social entre el alumnado puede favorecer que los estudiantes no tiendan a comparar sus resultados con sus compañeros (Buunk, Kuyper \& Van Der Zee, 2005). Asimismo, en línea con la literatura científica en el contexto de la EF (Barkoukis et al., 2014; Boiché et al., 2008), la desmotivación se relacionó negativamente con la calificación académica. Sin embargo, en este estudio la regulación externa parece no influir en los resultados académicos en la UD de salto con combas. Este hecho puede deberse al curso académico que compuso la muestra del estudio. Los sujetos de menor edad pueden no estar tan influenciados por los premios o castigos que el profesorado de EF establece (e.g., las calificaciones obtenidas) mostrando menores niveles de regulación externa (Ntoumanis, Barkoukis \& Thogersen-Ntoumani, 2009). Por otro lado, el aburrimiento también mostró una relación negativa con la calificación académica en el presente estudio. Desde el punto de vista educativo, este hecho debería ser tenido en cuenta ya que algunos autores han señalado que la relación negativa entre estas dos variables puede ser bidireccional. Es decir, que el aburrimiento puede generar un menor rendimiento académico y ese menor rendimiento, a su vez, puede dar lugar a un mayor aburrimiento (Pekrun, Hall, Goetz \& Perry, 2014). Todo ello hace que la utilización de estrategias motivacionales enfocadas a la reducción del aburrimiento resulten fundamentales en las clases de EF. Sin embargo, parecen necesarios más trabajos que refuten los resultados del presente estudio ya que el valor de las correlaciones no fue muy alto.

Los hallazgos encontrados sugieren que las calificaciones académicas obtenidas por el alumnado en las clases de EF pueden depender en gran medida del clima motivacional generado por el docente de EF, especialmente por el desarrollo de un clima motivacional tarea. En este sentido, parece necesaria la formación docente en estrategias motivacionales vinculadas a la aplicación de las áreas TARGET(Ames, 1992). Por ejemplo, si el docente de EF utiliza un sistema de evaluación en el que se valora el esfuerzo y el progreso personal inter-individual del alumnado por encima del resultado final, puede resultar más probable que se favorezca un clima de aprendizaje orientado a la tarea, y en consecuenciauna mejor calificación.

No obstante, debido al diseño adoptado por la investigación, este trabajo presenta una serie de limitaciones que se deben tener en cuenta. En primer lugar, se trata de un estudio de corte transversal, en solo una UD deEF, donde no existe una relación de causalidad entre las diferentes variables del estudio. Asimismo, a pesar de la rigurosidad seguida en el proceso de evaluación, las calificaciones pueden verse afectadas por elementos subjetivos fuera de nuestro control que podrían influir en las relaciones encontradas (Lekholm \& Cliffordson, 2009). Como prospectivas de futuro se abre la necesidad de seguir profundizando en esta línea de estudio, ya que existe un limitado número de trabajos en el contexto de la EF que analicen la relación del estilo interpersonal docente, y otras variables motivacionales, sobre la calificación de una UD y la calificación final de la asignatura. En esta línea, la implementación de estudios de intervención que analicen los efectos del clima motivacional tarea sobre la calificación obtenida por el alumnado se abren como una futura línea de estudio. De igual modo, parecen necesarios más estudios de corte longitudinal para analizar el efecto del clima motivacional tarea y ego en el rendimiento académico del alumnado.

\section{Conclusiones}

Este estudio ha reflejado la importancia de las variables motivacionales, especialmente el clima motivacional tarea generado por el docente de EF, en las calificaciones académicas obtenidas por el alumnado en una UD de salto con combas. Asimismo, parece importante evitar un clima motivacional ego ya que se relaciona, aunque en menor medida, con un menor rendimiento académico. Estos resultados sugieren que las estrategias motivacionales o métodos pedagógicos utilizados por el docente de EF podrían provocar que el alumnado perciba que su esfuerzo se evalúa en base a su progresión, y no a la comparación con sus compañeros, lo que podría conllevar unos mejores resultados académicos.

\section{Agradecimientos}

Agradecer la cofinanciación del Gobierno de Aragón y del Fondo Social Europeo.

\section{Referencias}

Alivernini, F., \& Lucidi, F. (2011). Relationship between social context, selfefficacy, motivation, academic achievement, and intention to drop out of high school: A longitudinal study. The Journal of Educational Research, 104(4), 
241-252. doi:10.1080/00220671003728062

Ames, C. (1992). Achievement goals, motivational climate, and motivational processes. In G. C. Roberts (Ed.), Motivation in sport and exercise (pp. 161176). Champaign, IL: Human Kinetics.

Baena-Extremera, A., Granero-Gallegos,A., Bracho-Amador, C., \& Pérez-Quero, F. J. (2012). Spanish version of the sport satisfaction instrument (SSI) adapted to physical education. Journal of Psychodidactics, 17(2), 377-395. doi: 10.1387/ Rev.Psicodidact.4496

Barkoukis, V., Taylor, I., Chanal, J., \& Ntoumanis, N. (2014). The relation between student motivation and student grades in physical education: A 3-year investigation. Scandinavian Journal of Medicine \& Science in Sports, 24(5), 406-414. doi:10.1111/sms.12174

Biddle, S. J., Cury, F., Goudas, M., Sarrazin, P. H., Famose, J. P., \& Durand, M. (1995). Development of scales to measure perceived physical education class climate: A cross-national project. British Journal of Educational Psychology, 65, 341-358.

Bobbit, S. (2003). Learning Environment, Motivation, and Achievement in High School Science. Journal of Research in Science Teaching, 40(4), 347-368.

Boiché, J. C. S., Sarrazin, P. G., Grouzet, F. M. E., Pelletier L. G., \& Chanal J. P. (2008). Students' motivational profiles and achievement outcomes in physical education: Aself-determination perspective. Journal of Educational Psychology, 100, 688-701.

Buunk, B. P., Kuyper, H., \& Van Der Zee, Y. G. (2005). Affective response to social comparison in the classroom. Basic and Applied Social Psychology, 27(3), 229-237.

Calero, J., Choi, A., \& Waisgrais, S. (2010). Determinantes del riesgo de fracaso escolar en España: una aproximación a través de un análisis logístico multinivel aplicado a PISA-2006. Revista de Educación, 225-256.

Cecchini, J. A., Fernández-Río, J., \& Méndez-Giménez, A. (2014). Effects of Epstein's TARGET on adolescents' intentions to be physically active and leisure-time physical activity. Health Educational Research, 29(3), 485-490. doi: 10.1093/her/cyu007

Coterón-López, J., Franco, E., Pérez-Tejero, J., \& Sampedro, J. (2013). Clima motivacional, competencia percibida, compromiso y ansiedad en Educación Física. Diferencias en función de la obligatoriedad de la enseñanza. Revista de Psicología del Deporte, 22(1), 151-157.

Deci, E. L., \& Ryan, R. M. (1985). Intrinsic motivation and self-determination in human behavior. New York: Plenum Press.

Duda, J. L., \& Nicholls, J. G. (1992). Dimensions of achievement motivation in scholwork and sport. Journal of Educational Psychology, 84(3), 290-299. doi: 10.1037/0022-0663.84.3.290

Fati-Ashtiani, A., Ejei, J., Khodapanahi, M., \& Tarkhorani, H. (2007). Relation between self-concept, selfsteem, anxiety, depression and academic achievement in adolescents. Journal of Applied Sciences, 7(7), 995-1000. doi:10.3923/ jas.2007.995.1000

Fernández-Río, J., Méndez-Giménez,A., \& Cecchini, J.A. (2014). A cluster analysis on students' perceived motivational climate. Implications on psycho-social variables. The Spanish Journal of Psychology, 17(18). doi: 10.1017/sjp.2014.21

Garcia, D., Jimmefors, A., Mousavi, F., Adrianson, L., Rosenberg, P., \& Archer, T. (2015). Self-regulatory mode (locomotion and assessment), well-being (subjective and psychological), and exercise behavior (frequency and intensity) in relation to high school pupils' academic achievement. PeerJ, 3, e847.

González, J., \& Portolés, A. (2014). Actividad física extraescolar: Relaciones con la motivación educativa, rendimiento académico y conductas asociadas a la salud. Revista Iberoamericana de Psicología del Ejercicio y el Deporte, 9(1), 51-65.

Guay, F., Vallerand, R. J., \& Blanchard, C. (2000). On the assessment of state intrinsic and extrinsic motivation: The situational motivation scale (SIMS) Motivation and Emotion, 24(3), 175-213.

Gutiérrez, M., Ruiz, L. M., \& López, E. (2011). Clima motivacional en Educación Física: concordancia entre las percepciones de los alumnos y las de sus profesores. Revista de Psicología del Deporte, 20(2), 321-335.

Huang, C. (2015). Academic achievement and subsequent depression: A metaanalysis of longitudinal studies. Journal of Child and Family Studies, 24(2), 434-442.

Jaakkola, T., Wang, C., Soini, M., \& Liukkonen, J. (2015). Students’ perceptions of motivational climate and enjoyment in Finnish physical education: A latent profile analysis. Journal of Sports Science and Medicine, 14(3), 477-483.

King, R. B. (2015). Sense of relatedness boosts engagement, achievement, and wellbeing: A latent growth model study. Contemporary Educational Psychology, 42, 26-38. doi:10.1016/j.cedpsych.2015.04.002

Legault, L., Green-Demers, I., \& Pelletier, L. (2006). Why do high school students lack motivation in the classroom? Toward an understanding of academic amotivation and the role of social support. Journal of Educational Psychology, 98(3), 567-582. doi:10.1037/0022-0663.98.3.567

Lekholm, A., \& Cliffordson, C. (2009). Effects of student characteristics and gender on grades in compulsory school. Educational Research and Evaluation, 15(1), $1-23$.

León, J., Nuñez, J., \& Liew, J. (2015). Self-determination and STEM Education: Effects of autonomy, motivation, and self-regulated learning on high school math achievement. Learning and Individual Differences, 43, 156-163. doi: 10.1016/j.lindif.2015.08.017
Ley Orgánica 2/2006, de 3 de mayo, de Educación (LOE). Publicada en BOE de 4 de mayo de 2006.

Liukkonen, J., Barkoukis, V., Watt, A., \& Jaakkola, T. (2010). Motivational climate and students' emotional experiences and effort in physical Education. Journal of Educational Research, 103(5), 295-308.

Martín, E., Martínez-Arias, R., Marchesi, A., \& Pérez, E. M. (2008). Variables that predict academic achievement in the spanish compulsory educational system: a longitudinal, multi-level analysis. The Spanish Journal of Psychology, 11, 400-415.

Martín-Albo, J., Nuñez, J. L., \& Navarro, J. G. (2009). Validation of the Spanish Version of the Situational Motivation Scale(EMSI) in the Educational Context. The Spanish Journal of Psychology, 12(2), 799-807.

Moreno-Murcia, J. A., Cervelló, E., Montero, C., Vera, J. A., \& García, T. (2012). Metas sociales, necesidades psicológicas básicas y motivación intrínseca como predictores de la percepción del esfuerzo en las clases de educación física. Revista de Psicología del Deporte, 21(2), 215-221.

Moreno-Murcia, J. A., Silveira, Y., \& Alias, A. (2015). Modelo predictivo para la mejora de la percepción de competencia y rendimiento académico en estudiantes universitarios. REDU. Revista de Docencia Universitaria, 13, 173-188.

Montero, I., \& León, O. (2007). A guide for naming research studies in Psychology. International Journal of Clinical and Health Psychology, 7, 847-862.

Moreno, J. A., González-Cutre, D., Chillón, M., \& Parra, N. (2008). Adaptación a la educación física de la Escala de las Necesidades Psicológicas Básicas en el Ejercicio (BPNES). Revista Mexicana de Psicología, 25(2), 295-303.

Moreno, B., Jiménez, R., Gil, A., Aspano, M. I., \& Torrero, F. (2011). Análisis de la percepción del clima motivacional, necesidades psicológicas básicas, motivación autodeterminada y conductas de disciplina de estudiantes adolescentes en las clases de Educación Física. Motricidad: Revista de Ciencias de la Actividad Física y del Deporte, 26, 1-24.

Nicholls, J. (1989). The competitive ethos and. democratic education. Cambridge, MS: Harvard University Press.

Niemiec, C. P., \& Ryan, R. M. (2009). Autonomy, competence, and relatedness in the classroom: Applying self-determination theory to educational practice. Theory and Research in Education, 7(2), 133-144.

Ntoumanis, N., Barkoukis, V., \& Thøgersen-Ntoumani, C. (2009). Developmental trajectories of motivation in physical education: Course, demographic differences and antecedents. Journal of Educational Psychology, 101(3), 717-728. doi: 10.1037/a0014696

Orden de 9 de mayo de 2007, del Departamento de Educación, Cultura y Deporte, por la que se aprueba el currículo de la Educación Secundaria obligatoria y se autoriza su aplicación en los centros docentes de la Comunidad autónoma de Aragón, 65, 8871-9024. B.O.A. 1 de junio de 2007.

Pekrun, R., Hall, N. C., Goetz, T., \& Perry, R. P. (2014). Boredom and academic achievement: testing a model of reciprocal causation. Journal of Educational Psychology, 106(3), 696-710. doi: 10.1037/a0036006

Reyes, M. R., Brackett, M. A., Rivers, S. E., White, M., \& Salovey, P. (2012). Classroom emotional climate, student engagement, and academic achievement. Journal of Educational Psychology, 104, 700-712. doi:10.1037/ a0027268

Risso, A., Peralbo, M., \& Barca, A. (2010). Cambios en las variables predictoras del rendimiento escolar en Enseñanza Secundaria. Psicothema, 22(4), 790-796.

Ryan, R. M., \& Deci, E. L. (2000). Self-determination theory and the facilitation of intrinsic motivation, social development, and well-being. The American Psychologist, 55(1), 68-78. doi: 10.1037110003-066X.55.1.68

Sternberg, R. J. (2010). Educational Psychology. Upper Saddle River, NJ: Pearson.

Stolz, S., \& Pill, S. (2014). Teaching games and sport for understanding: Exploring and reconsidering its relevance in physical education. European Physical Education Review, 20, 36-71. doi:10.1177/1356336X13496001

Ulstad, S. O., Halvari, H., Sørebø, Ø., \& Deci, E. L. (2016). Motivation, Learning Strategies, and Performance in Physical Education at Secondary School.Advances in Physical Education, 6, 27-41.

Urdan, T., \& Schoenfelder, E. (2006). Classroom effects on student motivation: Goal structures, social relationships, and competence beliefs. Journal of School Psychology, 44(5), 331-349. doi:10.1016/j.jsp.2006.04.003

Vlachopoulos, S. P., \& Michailidou, S. (2006). Development and initial validation of a measure of autonomy, competence, and relatedness in exercise: The Basic Psychological Needs in Exercise Scale. Measurement in Physical Education and Exercise Science, 10(3), 179-201. doi: 10.1207/s15327841mpee1003_4.

Wang, M., \& Holcombe, R. (2010). Adolescents' perceptions of classroom environment, school engagement, and academic achievement. American Educational Research Journal, 47, 633-662. doi:10.3102/0002831209361209

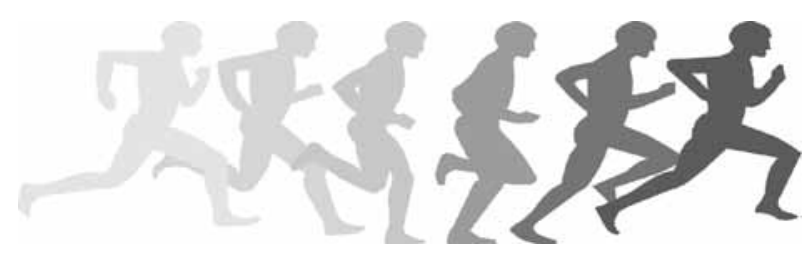

